\title{
Asymptomatic left isomerism with preduodenal portal vein: computed tomography appearance
}

\author{
Bruno Coulier ${ }^{1}[$
}

Received: 22 February 2021 / Accepted: 3 April 2021 / Published online: 13 April 2021

(c) The Author(s), under exclusive licence to Springer-Verlag France SAS, part of Springer Nature 2021

\begin{abstract}
Left isomerism (polysplenia), one of the two major variants of heterotaxia with right isomerism (asplenia), may be rarely diagnosed in adulthood. Most cases are nevertheless asymptomatic and incidentally detected during imaging or surgery performed for unrelated conditions. We hereby report a case of left isomerism fortuitously diagnosed in a 55-year-old man with unrelated tachy-cardiomyopathy. Thoraco-abdominal computed tomography revealed a typical preduodenal portal vein (PDPV) associated with a large series of other occult anatomic variations comprising: polysplenia, agenesis of both pancreatic body and tail, complete non-rotation of the bowel and finally azygous continuation of the inferior vena cava. Subtle but highly specific thoracic features of left isomerism were also found with a bilobed right lung and bilateral long hyparterial main bronchi. The features of adult left isomerism are remembered with special attention to the PDPV.
\end{abstract}

Keywords Heterotaxia syndrome $\cdot$ Left isomerism $\cdot$ Polysplenia $\cdot$ Preduodenal portal vein $\cdot$ IVC abnormality $\cdot$ Pancreatic agenesis $\cdot$ Situs ambiguous $\cdot$ Intestinal malrotation

\section{Introduction}

During embryology, a series of thoraco-abdominal unpaired organs follow a specific spatial organization. There are typical right-sided structures (liver, superior and inferior vena cava, right atrium and its appendage, trilobed right lung with epi-arterial bronchus, etc.) and typical left-sided structures (stomach, spleen, left atrium and appendage, pulmonary veins and bilobed left lung with hyparterial superior lobar bronchus, etc.).

The normal final positioning refers to situs solitus (with thoracic levocardia) and the complete mirror-image arrangement refers to situs inversus, a rare but commonly asymptomatic situation [6].

Situs ambiguous, more commonly also named heterotaxia refers to anatomic arrangements in which the major organs are distributed abnormally within the chest and/or abdomen $[2,5,6]$. This kind of variation may be isolated or associated with an extensive collection of complex thoracic and/or abdominal anomalies [6]. The prevalence of HS is

Bruno Coulier

bcoulier.md@gmail.com

1 Department of Diagnostic Radiology, Clinique St Luc, Bouge, 5004 Namur, Belgium estimated to be 1 in 10,000 people worldwide but the condition appears probably largely underdiagnosed. There are two main variants of heterotaxia: left isomerism (LI) also known as polysplenia or bilateral left sidedness and right isomerism (RI) also known as asplenia or bilateral right sidedness $[2,5,6]$.

Clinical expression of HS variants depends on the gravity of the abnormalities involved. HS may be life threatening in infancy or childhood in a high proportion of cases. This is essentially due to severe cardiac abnormalities particularly in the RI variant [6]. Rare individual adults, especially having an LI, may nevertheless present only mild health disturbances or may be completely asymptomatic. We hereby report a case of LI fortuitously diagnosed in a 55-year-old man with unrelated tachy-cardiomyopathy.

\section{Case report}

A 55 -year-old patient $(144 \mathrm{~kg} / 1.7 \mathrm{~m})$ was admitted with complaints of dyspnea, abdominal ascitic distention, anasarca and progressive worsening of oedema of the lower limbs. In the health context of COVID-19, thoraco-abdominal computed tomography (CTA) was performed not only to exclude COVID-19 pneumonia and/or pulmonary embolism 
but also to evaluate the etiology of ascitis. Pulmonary embolism was excluded but a large azygous continuation of the inferior vena cava (of which only a short supra-hepatic segment remained visible) was seen (Fig. 1a-c). A large collection of abdominal anatomic variations was also found comprising: an unusual preduodenal portal vein (PDPV) (Fig. 1d, e), two spleens nested one inside the other (polysplenia), the absence of pancreatic body and tail (Fig. 2a, b), a complete non-rotation of the bowel (Fig. 2c), an undescended epigastric cecum with an epigastric appendix (Fig. 2d). Scrupulous retrospective analysis of the thoracic CT revealed a bilobed right lung (Fig. 2f) with an atypical " $z$ "-shaped scissure and bilateral hyparterial long main bronchi (Fig. 2g, h).

The patient was finally diagnosed having a completely asymptomatic LI (polysplenia). It was a fortuitous diagnosis superimposing on symptomatic cardiac decompensation caused by impaired systolic function and atrial fibrillation, both generating secondary tachy-cardiomyopathy. The patient was successfully medically treated.

\section{Discussion}

LI and RI are the main variants of HS. Unlike situs abnormalities leading to ordinary or reverse lateralization, isomerism is accompanied by an atypical anatomy with symmetrisation of the organs supposed to be lateralized or even duplication of organs in certain circumstances $[2,5,6]$.

$\mathrm{RI}$ is the most frequently variant observed at birth. In this situation, there are two atria of the systemic right type and the two lungs of the right type. Both lungs are thus trilobed with bilateral minor fissures and their main bronchi are short and located superior to the ipsilateral main
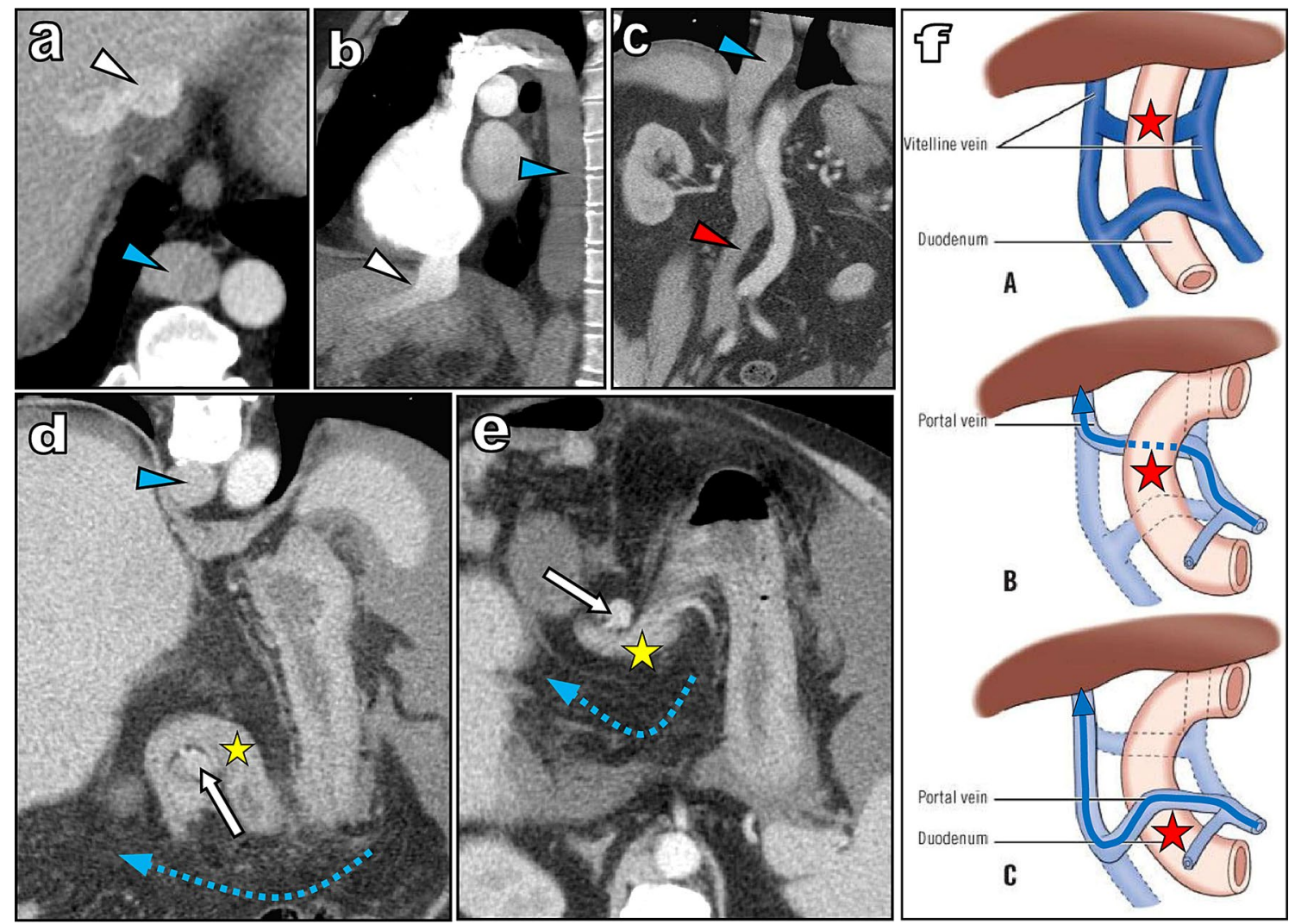

Fig. 1 On angio-computed tomography (CTA) performed to exclude pulmonary embolism, axial a and sagittal $\mathbf{b}$ views shows only a short sus-hepatic segment (white arrowhead) of inferior vena cava (IVC); the main infra-hepatic IVC is interrupted with azygous continuation (blue arrowhead); coronal view $\mathbf{c}$ of complementary abdominal CT (performed to investigate ascites) confirms the direct continuation of the IVC (red arrowhead) by the great azygous vein (blue arrowhead); epigastric coronal oblique $\mathbf{d}$ and axial oblique e views clearly identify an abnormal preduodenal portal vein (PPV) (white arrow); the gastroduodenal junction (yellow star) wraps posteriorly around this PPV; the greater omentum unfolds below the gastro-duodenoportal complex (red curved dotted arrow). Schematic representation (f) of the embryologic development of the vena porta: A) the right end left vitelline veins frame the primitive gut (red star) with their middle posterior and caudal anterior communicating segments; B) in normal development, the vitelline veins loss their right caudal, left cranial and the anterior caudal communicating segments resulting in the development of a normal ' $\mathrm{S}$ '-shaped portal vein; C) PDPV occurs when the primitive vitelline veins rather loss their left crania, right distal and middle posterior communicating segment 

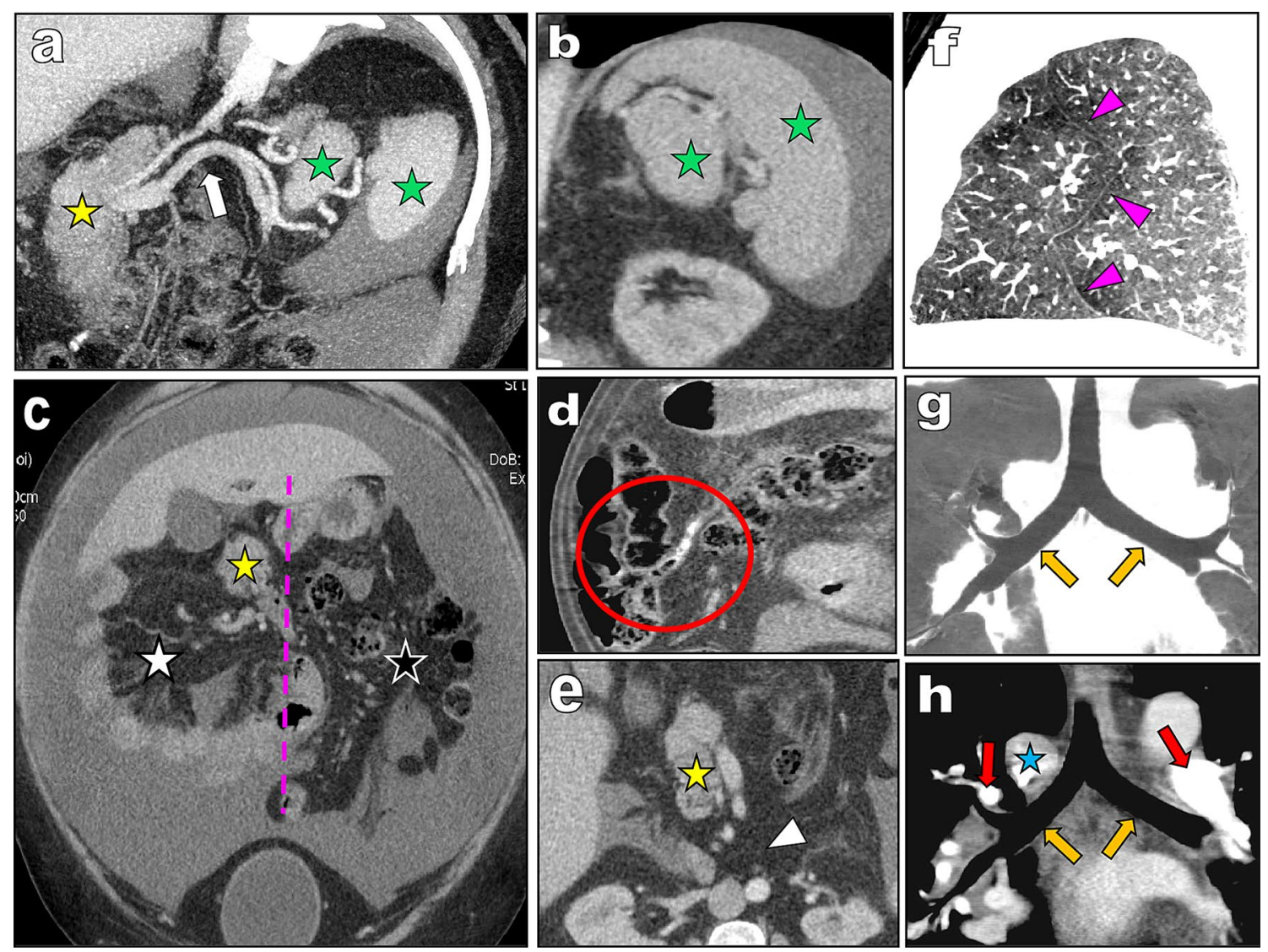

Fig. 2 Further examination of the patient's abdominal CT; coronal oblique maximal intensity projection a and coronal oblique view b show: two spleens nested one inside the other (green stars) and absence of both pancreatic body and tail; the consequences are "naked" splenic vessels (white arrow). Global coronal view c shows massive ascites gathering the hollow viscera in the midline of the abdomen; any mesenteric or mesocolic fixation in the posterolateral gutters are absent; the small bowel is on the right and the colon is on the left (non-rotation) of the midline (pink dotted line); $\mathbf{d}$ the undescended cecum is seen anteriorly in the middle of the epigastrium and the appendix is sagittal and posteriorly oriented (red circle); epigastric axial view e shows the isolated sagittal pancreatic head (yel-

pulmonary artery (epi-arterial bronchi). Asplenia is associated in $80 \%$ of cases. The liver may be centrally located and the stomach in an indeterminate position. Major and very severe cardiac abnormalities are associated in nearly $100 \%$ of patients (transposition of the great vessels, tetralogy of Fallot or atrioventricular duct type...) determining a very poor neonatal prognosis. Survival beyond 1 year of life is rare, ranging from less than 5-20\%. RI may rarely exist without cardiac anomalies and may exceptionally remain asymptomatic until adulthood [2, 5-8].

In LI as reported in this case, the lungs are of the left type. They are thus bilobed and their main bronchi are long, passing inferiorly to the ipsilateral main pulmonary artery PA (hypo-arterial bronchi). LI is classically associated with both atria of the pulmonary type, a midline liver. A high low star); there is no angle of Treitz and the preaortic third duodenum is absent (white arrowhead). Scrupulous retrospective review of the thoracic CTA reveals a bilobed right lung $\mathbf{f}$ with an atypical " $\mathrm{z}$ " shaped scissure. The "anterior" lobe regroups the middle lobe and the anterior segment of the upper lobe; the "posterior" lobe regroups the apex of the upper lobe and the lower lobe. Coronal minimal intensity projection $\mathbf{g}$ and coronal view $\mathbf{h}$ show that the main bronchi (orange arrows) are long and pass inferiorly to the ipsilateral main pulmonary artery (PA) (red arrows); these bronchi are "hyparterial", the usual conformation of the left lung. Arch of the hypertrophied azygos vein $=$ blue star

prevalence of polysplenia is typical and explains the former but still used term of "polysplenia". Cardiac abnormalities are less common and less complex than in RI. As a consequence, the death rate is lower (60\% in the first year) than in RI. Only about $10 \%$ of patients may reach adulthood without any complications.

At bird, LI isomerism is more common in females but the exact incidence and sex ratio in adults is more difficult to estimate because, as is the reported case, many adult cases are only incidentally detected during imaging evaluation for unrelated conditions. Minimal cases presenting with only bilobed lungs but a normal abdominal situs and without cardiac abnormality have also been reported [1, 2, 5-9]. LI may also present with other variable symptoms related to a large spectrum of other associated variations. There is no single 
pathognomonic abnormality that characterizes LI [1]. In the abdomen, polysplenia, a short pancreas, abnormalities of the portal vein formation, various types of gut malrotations and inferior vena cava anomalies are the most common associated anomalies [1, 2, 7-9] apart from the specific thoracic variations:

- Polysplenia is characterized by the presence of several spleens, usually between two and six. They are about of equal size, associated with the main spleen in the left hypochondrium and vascularized by an artery with normal birth. Polysplenia must be differentiated from supernumerary spleens (which generally measure only $1-2 \mathrm{~cm}$ ) and from splenosis [3].

- Congenital preduodenal portal vein (PDPV), often present (75\%) in LI, is characterized by a portal vein passing in front of the duodenum [3,7-11]. PDPV is rare as an isolated variation $(25 \%)$ [4, 11]. In childhood, PDPV may be associated with features of intestinal obstruction caused by coexisting congenital anomalies such as duodenal atresia, duodenal web, annular pancreas, intestinal malformation, biliary atresia, etc. [7]. Surgery may be required for these obstructive conditions. Duodenal obstruction caused by PDPV alone is a very rare situation with only a few reported cases. The reason is probably the fact that PDPV is a thin-walled vessel under low pressure $[3,11]$. As reported in our case, patients with PDVP may reach adulthood without any symptoms, the anomaly being often found during examinations or surgery for unrelated conditions. PDPV develops between the 5th and 10th embryonal weeks [11] when the paired primitive vitelline veins loss their left cranial, right distal and middle posterior communicating segments (Fig. 2fb). In normal development, the normal ' $\mathrm{S}$ '-shaped retroduodenal portal vein rather results from the loss of the right caudal, left cranial and anterior caudal communicating segments of the vitelline veins (Fig. 2fc) [8]. An occult PDPV not detected prior to biliary surgery may be responsible of severe complications, such as hemorrhage or accidental vascular ligation. Such accidents can be prevented by performing previous careful diagnostic imaging and especially by searching and noting the possibility of PDPV in cases of polysplenia syndrome.

- In $25 \%$ of cases, but not in this case, a median liver is present in LI (but also in RI) with two symmetrical hepatic lobes and a gallbladder interposed between them [3].

- Variations of the digestive tract associated with LI may comprise an intraperitoneal annular pancreas or sometimes, as illustrated in our case, a short pancreas with agenesis of the corpus and tail [3, 8]. Pancreas formation results from fusion of ventral and dorsal pancreatic buds. The ventral bud gives rise to the head and unci- nate process, while the dorsal bud gives rise to the body and tail. The development of pancreatic dorsal bud and spleen occurs in the dorsal mesogastrium [3, 8, 9]. As a consequence, simultaneous anomalies in both these organs can be expected in LI (polysplenia). Agenesis of the dorsal bud could be related to a defect of blood supply during intrauterine life caused hemodiversion secondary to the presence of polysplenia [3].

- Al though not pathognomonic of LI, inferior vena cava interruption with azygous or hemiazygos continuation, as clearly found in our patient, is the most common anatomic finding of LI (polysplenia) [1]. The short intrahepatic segment of the inferior vena cava is nevertheless present in these cases.

- Malrotation of the bowel is a frequent finding in LI or RI [1]. The prevalence may approach $70 \%$. This high prevalence predisposes patients to volvulus. Cases have been reported with normal rotation, incomplete rotation, non-rotation (as reported in our case) and even with the rarest form of reverse rotation [1].

Due to the large range of possible combinations of various malformations and the overlapping spectrum of findings commonly seen in LI and RI, a segmental or individualized approach is now preferred. This approach consists in noting and describing all malformations present in each individual patient $[1,2]$.

\section{Conclusion}

Although more frequent than RI, LI presenting in adulthood remains very rare, mainly asymptomatic and often discovered incidentally in patients imaged or operated for unrelated reasons. The presentation may vary from one single anatomical variation to a large cluster of thoracic and abdominal variations. Radiologists who are confronted with this kind of rare syndrome must be very careful. Surgical complications may arise from any unrecognized anomalies such as PDVP or intestinal malrotation.

The demonstration of variation such as a PDVP should be followed by careful evaluation for any other frequently associated individual abnormalities.

Author's contributions Bruno Coulier is the first author and corresponding author of the manuscript. Bruno Coulier performed the imaging studies, collected and managed the data, wrote the manuscript.

Funding The author did not receive support from any organization for the submitted work. 


\section{Declarations}

Conflict of interest The author declare that he has no conflicts of interest concerning this article.

Ethical approval The manuscript follows the prescriptions of the institutional ethical board. The author certify that all data and figures have been completely anonymised.

\section{References}

1. Applegate KE, Goske MJ, Pierce G, Murphy D (1999) Situs revisited: imaging of the heterotaxy syndrome. Radiographics 19:837-852 (discussion 853-4)

2. Desir A, Ghaye B (2009) Congenital abnormalities of intrathoracic airways. Radiol Clin North Am 47:203-225

3. Elhattabi K, Elyamine O, Bouali M, Abdelilah El Bakouri A, Fatimazahra Bensardi F, Fadil A (2020) Polysplenia with agenesis of the dorsal pancreas and preduodenal portal vein, about a case. Int J Surg Case Rep 76:357-360

4. Höing K, Ringe KI, Bektas H, Klempnauer J, Jäger MD (2015) Preduodenal superior mesenteric vein and Whipple procedure with vascular reconstruction-a case report. Int J Surg Case Rep 10:107-110
5. Kim S-J (2011) Heterotaxy syndrome. Korean Circ J 41:227-232

6. Lagrotta G, Moises M (2020) Heterotaxy polysplenia syndrome in adulthood: focused review and a case report. Cureus 30(12):e6822

7. Latha GA, Kagali NA, Shridhar M, Satish Prasad BS (2014) Preduodenal portal vein in adult with polysplenia syndrome revisited with a case report. Indian J Surg 76:137-142

8. Low JP, Williams D, Chaganti JR (2011) Polysplenia syndrome with agenesis of the dorsal pancreas and preduodenal portal vein presenting with obstructive jaundice-a case report and literature review. Br J Radiol 84(1007):e217-e220

9. Rameshbabu CS, Gupta KK, Qasim M, Gupta OP (2015) Heterotaxy polysplenia syndrome in an adult with unique vascular anomalies: case report with review of literature. J Radiol Case Rep 9:22-37

10. Seo H-I, Jeon TY, Sim MS, Kim S (2008) Polysplenia syndrome with preduodenal portal vein detected in adults. World J Gastroenterol 7(14):6418-6420

11. Shukla RM, Mukherjee PP, Mukhopadhyay B, Mandal KC (2013) Congenital duodenal obstruction with preduodenal portal vein and situs inversus totalis: report of two cases and literature review. Indian J Surg 75:74-76

Publisher's Note Springer Nature remains neutral with regard to jurisdictional claims in published maps and institutional affiliations. 